\title{
System for Detection of Vital Signals with an Embedded System \\ A.Maheswari ${ }^{1}$ and V.Ramachandran ${ }^{2}$ \\ ${ }^{1}$ Anna University, Chennai - 600025, ${ }^{2}$ Anna University, Chennai - 600025, India \\ maheswari.a@gmail.com
}

\begin{abstract}
Rapid advancement in the field of Embedded Systems and Wireless communications has permitted development of Revolutionary Medical Monitoring Systems and thus improving the lifestyle of patients. The system captures and analyzes the ECG signals in real time through a low cost embedded development board. The system can detect cardiac abnormalities with high precision. One of the objectives at the time of building the proposed system has been to optimize the resources, memory size and communication costs.
\end{abstract}

Keywords: electrocardiogram, LABVIEW, RCM 3700, real time webpage

\section{Introduction}

One of most pressing matters today in medical care is the response time to 'patients in need'. The "massive cardiac arrest” is generally considered as an unpredictable and unpreventable event.
Lot of patients die due to cardiac arrest. These cardiac abnormalities occur suddenly without a shred of warning. Recent studies show that there are generally significant cardiovascular abnormal symptoms such as palpitations, faints, chest pain, and shortness of breath before the sudden occurrence of cardiac arrest. If these abnormal symptoms are detected and diagnosed prior to the occurrence of heart attack, then the fatal caused by cardiac arrest can be prevented to a major extent. Therefore it is necessary to have an effective method for early detection of the abnormalities.

This paper proposes a real time monitoring system for detecting cardiac arrest and automatically alerting the dangerous abnormality through a low cost development kit RCM 3700 using electrocardiogram signals. The proposed system is more reliable and flexible as it aids persons with cardiovascular symptoms to monitor their heart status and 
detect abnormalities in their normal daily life.

\section{Current Research and Development} Activities

Traditionally Holter recording has been used during normal patient activity to record ECG signals. A Holter is a small, mobile and light device that records, during a period of 24, 48 hours or 72 hours, ECG signals which are later analyzed in the hospital. In HOLTER recording system, the ECG signals are processed later by dedicated software and then a diagnostic report will be created to aid cardiologists for further analysis. However, HOLTER is proved largely insufficient for a long-term prediction because the critical cardiac abnormalities do not necessarily occur during these 72 hours. The serious drawback of the system is that if the patient suffers from a serious rhythm, the Holter only records it, but it does not react to it in real time. This system does not detect the abnormalities of cardiac arrest when patients are out of the hospitals. Moreover, such a system cannot automatically transmit information at the moment when abnormal cardio activity is present.
Over a period of time, several research works are being carried out with a specific aim to build platforms for real time remote monitoring. Those systems perform some local real-time monitoring in order to detect some anomalies and send alarms to a control centre. In spite of the advantages, these systems present main problems related to the fact that a complete analysis is not performed in the place where the signal is acquired. Wireless communications imply a high cost and in case the wireless network is not available (e.g. in a tunnel, in an elevator, etc.) at some moment, there might be a loss of cardiac signal with the corresponding risk of not detecting some anomalies.

A small wireless ECG with bluetooth communication to a PDA was developed to monitor the cardiac signals in the year 2006. In this system, a small sized ECG sensor was connected to a handheld device that graphically presents the ECGsignals. A prototype for an embedded ECG sensor has been developed. Using bluetooth technology, the ECG sensor can connect to a personal digital assistant (PDA). This system can be made to work with ZigBee radio instead of the bluetooth, 
as it may resolve the problems with serial port communications.

A cellular phone based online cardiac signal processing system for ambulatory and continuous detection has been developed in 2007. It aids cardiovascular disease patients to monitor their heart status and detect abnormalities in their normal daily life. This system is a solution to the limitations in conventional clinic examination such as the difficulty in capturing rare events, off-hospital monitoring of patients' heart status and the absence of immediate dissemination of physician's instruction to the patients. However the processing of cardiac signals can be done only in mobile phone Dopod 595 windows mobile enabled phone with a Samsung $300 \mathrm{MHz}$ processor and it features the ARM5 instruction architecture set and on an O2 XphoneII. The O2 Xphone II has T1 OMAP 730 processor and features the ARM4 instruction set architecture.

\section{Acquisition of Electrocardiogram Signals}

In this research work, a biomedical kit bundled with LabVIEW is used to simulate ECG Signal. The configuration settings for ECG signal simulation are made as shown in fig.1.

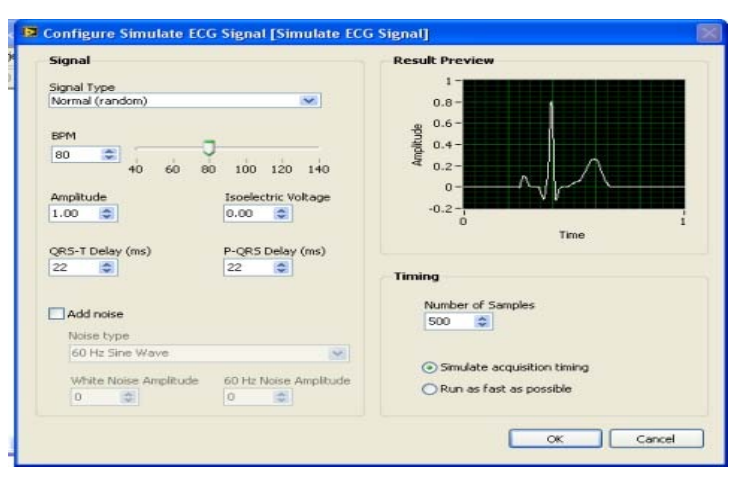

Fig. 1 Parameter selection window for cardiac signal simulation

Data acquisition has been coordinated using a computer system programmed in LABVIEW 8.5. This real time software allows a wide range of customizable settings, including minimum heart rate and maximum beats per minute. In LABVIEW, user interface is known as front panel and we can add code using icons of functions to control the front panel object. The block diagram contains the requisite code. The important factor in the data acquisition is the rate at which measuring device samples the incoming signal. In this study, the data acquisition is done at the rate of 500 samples per second. LABVIEW has provision for acquisition 
and saving the data. This yields more flexibility to the cardiologist.

\section{Detection of abnormalities in cardiac} signals using Embedded Development

\section{Board RCM 3700}

In this research work, RCM 3700 embedded development board with dynamic C, Integrated Development Environment has been used as a diagnosis tool. The basic architecture of the proposed system is shown in fig.2. It consists of three units namely, Data Acquisition Unit, Data Processing Unit and Data Communication Unit.

Data Acquisition Unit

ECG Signal simulation and acquisition

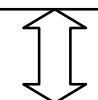

Data Processing Unit

RCM3700 Embedded Development board for cardiac signal conversion and further processing

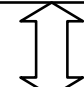

Data Communication Unit

Display of results through Embedded Web Server ported in R3000 of RCM3700 development board

Fig 2. Basic Architecture of the proposed System

\section{Data Acquisition Unit:}

The ECG data acquisition is coordinated in the host system programmed in LabVIEW 8.5. This real time software allows a wide range of customizable settings, including minimum and maximum heart rate. In fig. 2 the first block represents the ECG signal simulation and acquisition. The ECG signals are simulated by using the biomedical kit in LabVIEW software. The sampling rate of ECG signals in the proposed system is selected as 500 samples per second to include all the details of the signal. LabVIEW has provision to start acquisition and save data that gives more flexibility to the cardiologist. The simulated ECG signals are acquired by the embedded development board RCM 3700 through GPIB.

\section{Data Processing Unit:}

The data processing block in fig.2 contains the embedded development board and the local network created with RCM3700 module. The RCM3700 module is to the Prototyping Board. The programming cable is between the RCM3700 and the COM1 port in the 
workstation PC. The power supply delivers $7.5 \mathrm{~V}-30 \mathrm{~V} \mathrm{DC}$ at $500 \mathrm{~mA}$ for complete downloading of the application program into the target board.

The dynamic C Integrated Development Environment uses a COM (serial) port to communicate with the prototyping development board. As shown in fig. 3 , the acquired signals from the real time software in the data acquisition unit is given through the COM1 port in the host PC through the analog input port in the low cost development kit RCM3700 . Dynamic C is an integrated development environment for writing embedded software. The application program developed in dynamic $\mathrm{C}$ is downloaded into the embedded system using this programming cable. As illustrated in fig 4, a local network is created through a crossover cable between the host PC and RJ45 in the RCM3700 embedded development board.

\section{Results and Discussion}

The LabVIEW 8.5 simulator is used to simulate signals which eliminate the inconvenience of having a live testing subject. The real time software simulates the ECG signal, displays it onto the screen, and then sends them via the GPIB cable to RCM3700 target board for processing on the ECG signal data. The cardiac signals simulated from LABVIEW are shown below. The proposed system is designed to process two of the worst conditions of cardiac arrest namely, atrial tachycardia and hyperkalemia. Atrial tachycardia occurs when bpm (beats per minute) is greater than 100. Hyperkalemia occurs when the beats per minute is less than 60 .

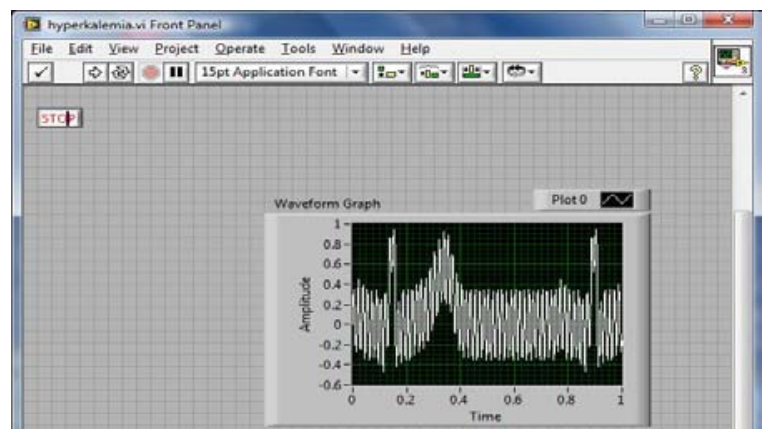

\section{Hyperkalemia}

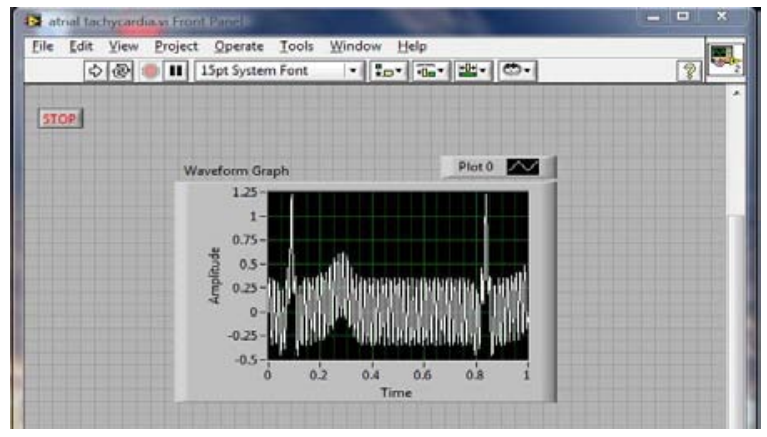

Atrial Tachycardia

Fig 3. Front panels in cardiac signal simulation 
The complete schematic of the proposed system is shown in the fig. 4 below

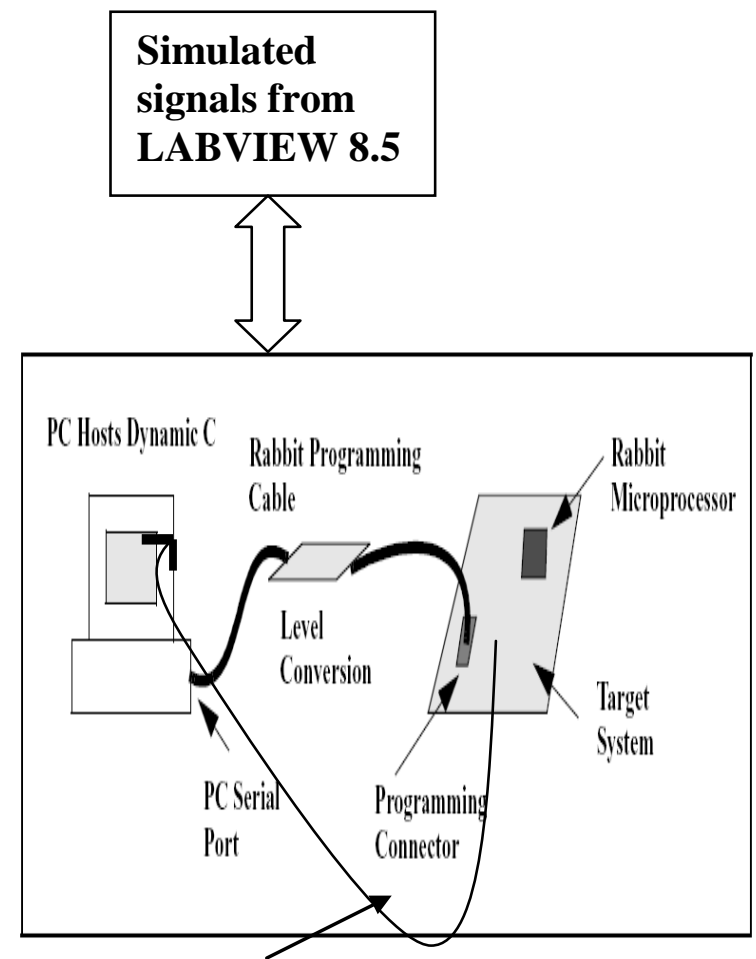

Processed results send as Web pages to Host PC

Fig 4. Schematic of the proposed system

The steps to download the application program into the target board are:

1. The initial loader, COLDLOAD. BIN, is loaded via "triplets" sent at 2400 bps from the PC to a target in bootstrap mode.

2. The COLDLOAD.BIN is run to load the secondary loader, pilot BIOS, at 57,600 bps (19,200 bps before Dynamic C 7.05).
3. The pilot BIOS, PILOT . BIN, and load the BIOS (as Dynamic C compiles it) are run.

\section{Data Communication Unit:}

The software for medical diagnosis is developed in dynamic C. In dynamic C Integrated Development Environment, the communication between the host PC and the target system is established serially. The baud rate of 115200 is selected for proper downloading of the application program into flash in the target system. The application program is compiled and downloaded from the host PC into flash in the target system. The results are displayed as Web pages in the host PC.

The RCM3700 development board module uses HTTP functions and structures in dynamic $\mathrm{C}$ to serve the Web page on request. The HTTP server uses HttpType and HttpSpec structures. These contain information relating to the files the Web server serves. The main () function in the application on the diagnosis of cardiac arrest begins by declaring variables and calls sock_init () to initialize the TCP / IP stack and calls the http_init () to initialize the embedded Web server. 
When the embedded Web server receives the service request from the browser, it firstly submits the request to the CGI program, and CGI program acquires the signals. The CGI program is executed in real time, so that it can output dynamic information. The Dynamic C's HTTP server and SHTML handler code manage the serving of the Web page, including accepting requests to connect, returning the requested pages and closing connections. After the code is compiled into the target board, the Web page can be accessed with the IP address of the board. The browser is made to point to http://10.10.1.100 . The fig. 5 and 6 show the result as Web pages in the host PC. The LED DS1 represents the condition, hyperkalemia and DS2 represents the condition, atrial tachycardia. When the RCM 3700 module runs this program, any computer that can access the module over the network can request the Web page and view the results.

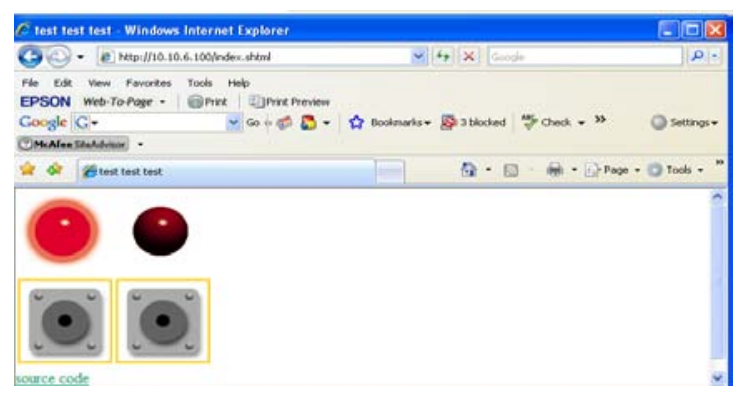

Fig. 5 Hyperkalemia

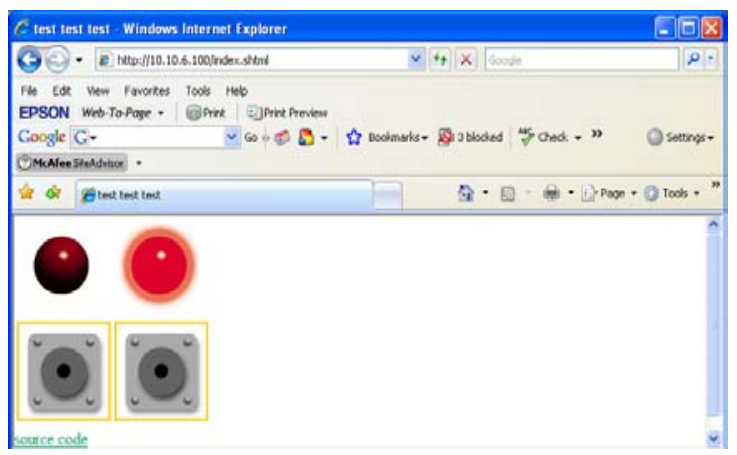

Fig. 6 Atrial Tachycardia

\section{Conclusion}

The proposed system provides a good solution for ambulatory and continuous monitoring when people are out-of-hospital and are in their daily life. The proposed system helps to detect abnormality early when no symptoms are present and prompt them for a medical check-up so that they can arrange for therapy as early as possible. In this system, the cardiac arrhythmia events can be detected and sent in real-time to the cardiologists. The length of time between onset of the event and delivery of treatment is reduced very much and hence time is considered as the most important determinant in the survival of victims who suffer a cardiac arrest. The survival rate gets increased. The proposed system has a huge potential to revolutionize the way healthcare is delivered throughout the world. 


\section{References}

1. Sridhar Vasudevan and K.J Cletus, 'Secure Telemedicine System for home health care', West Virgina University, Morgantown, IEEE, pp. 39-40.

2. Bentham.J: TCP / IP Lean - Web Servers for Embedded Systems

3. Igor Klimchynski, "Extensible Embedded Web Server for Internet Based Data Acquisition and Control", IEEE. 2004; p.nos:52 - 55

4. Miguel Dominguez, “A Simple Architecture for Embedded Web Servers”, Universidade do Minho, 4710 057 Braga, Portugal, and ICCA'03, p.nos:113-120

5. I.Agranat, "Embedded Web Servers in Network Devices”, Communication Systems design, March 1998, pp. 30-36

6. Tao Lin, Hai Zhao, Jiyong Wang, Guangjie Han, Jindong Wang "An Embedded Web Server for Equipments"

7. Janne Riihijarvi, Petri Mahonen and Mika J.Saaranen, University of Oulu, Jussi Roivainen and Juha-Pekka Soininen,
VTT Electronics, "Providing Network Connectivity for Small Appliances: A Functionally Minimized Embedded Web Server”

8. Patrick Gustafson, Jimmie Hagblad, "Miniaturised multi channel wireless ECG connected to graphical computer interface using Bluetooth", The Department of Computer Science and Electronics, Mälardalen University

9. V.Kumar, K. M.Adhikari, Y. D.

Singh "Fighting cardiac arrest: Automated external defibrillator”, Indian Society of Critical Care Medicine, ESTD. 1993

10. Arnold Baruch Simon, Stuart, FL, US, "Method and Device for detecting cardiac arrest and automatically alerting emergency personnel of wearer's location”, United States Patent Publication, September 2003

11. Meng Jiang, “Tele-cardiology Sensor Networks for Remote ECG Monitoring”, Dept. of Computer Engineering, Rochester Institute of Technology Rochester, NY, October, 2006 
12. X Chen, CT Ho, ET Lim, "Cellular Phone Based Online ECG Processing for Ambulatory and Continuous Detection”, TZ Kyaw Institute of Infocomm Research, SG, Singapore, Computers in Cardiology 2007; 34:653-65

13. ECG library from Physionet, http://www.ecglibrary.com/ "LABVIEW User Manual” from National Instruments

14. Yanzheng LI, Shuicai WU, Jia LI, Yanping BAI, "The ECG Telemonitor Based on Embedded Web Server", Biomedical Engineering Center, Beijing University of Technology Beijing China, page nos: 752-755

15. Haiying Zhou, Kun Mean Hou, Laurent Gineste, Christophe De Vaulx, "A New System Dedicated to Real-time Cardiac Arrhythmias Tele-assistance and Monitoring”, Journal of Universal Computer Science, vol. 12, no. 1 (2006), 30-44

16. David J. Christini, "Using RT-Linux for Adaptive Cardiac Arrhythmia Control", Division of Cardiology, Weill Medical College of Cornell University, New York
17. Jan Axelson, "Embedded Ethernet and Internet Complete”, Published by Lakeview research, 2003

18 X Chen, CT Ho, ET Lim, TZ Kyaw, “Cellular Phone Based Online ECG Processing for Ambulatory and Continuous Detection" Institute of Infocomm Research, SG, Singapore 